\title{
EL PAPEL DE LA REPETICIÓN Y EL RENDIMIENTO ACADÉMICO EN EL ABANDONO ESCOLAR TEMPRANO
}

\author{
Lucía Roldán Prego \\ Universidade da Coruña, A Coruña, España \\ lucia.roldan@udc.es \\ Carolina Rodríguez Llorente \\ Universidade da Coruña, A Coruña, España \\ carolina.rodriguez.llorente@udc.es \\ Tania Vieites Lestón \\ Universidade da Coruña, A Coruña, España \\ t.vieites@udc.es
}

Fecha de Recepción: 5 Julio 2019

Fecha de Admisión: 25 Septiembre 2019

\section{RESUMEN}

Dadas las graves consecuencias negativas de abandonar la escuela para el individuo y para la sociedad, los investigadores han tratado de identificar los factores que predicen el abandono con la esperanza de desarrollar intervenciones que mejoren los procesos de riesgo. Teniendo en cuenta las dificultades para medir y evaluar los factores que afectan a los abandonos que ya han ocurrido y asumiendo la relación entre la intención de abandono y el abandono real, parece esencial desarroIlar una caracterización amplia de los estudiantes que tienen la intención de abandonar. En este punto, con una muestra de 267 alumnos españoles (48.7\% mujeres; $M_{a g e}=14.38 ; S D=1.09$ ) que cursaban $2^{0}(n=138)$ y $3^{\circ}(n=129)$ de ESO se explora la incidencia de la repetición y el rendimiento académico sobre la intención de abandonar antes de completar la educación secundaria obligatoria. Los resultados de este trabajo sugieren que la probabilidad de que se dé una intención de abandono temprano es significativamente más alta entre quienes han repetido uno o dos cursos en la ESO que entre aquellos que no han repetido en ESO ( $O R=8,769$, IC $95 \% 2,304-33,378 ; Q=.79)$ y que la probabilidad de abandonar puede ser también más elevada entre los estudiantes que informan de rendimiento académico inferior a la media que entre quienes informan de rendimiento superior a la media ( $O R=4,336$, IC 95\%,968-19,421; Q=.64). Atendiendo a nuestros resultados, si bien no estar repitiendo actualmente o no haber repetido en EP podría garantizar, en cierta medida, la intención de completar los estudios, ni estos factores ni las bajas calificaciones se establecen como predictores directos de la intención de abandono temprano. A la luz de estos resultados cabe seguir explorando el perfil personal y motivacional del abandono escolar temprano.

Palabras clave: abandono escolar temprano; repetición de curso; rendimiento académico; intención de abandono. 


\title{
EL PAPEL DE LA REPETICIÓN Y EL RENDIMIENTO ACADÉMICO EN EL ABANDONO ESCOLAR TEMPRANO
}

\begin{abstract}
The role of grade repetition and academic achievement in early school dropout. Given the serious negative consequences of dropping out of school for both the individual and society, researchers have tried to identify the factors that predict students' drop out, in the hope of developing interventions that improve risky processes. Taking into account the difficulties in measuring and assessing the factors affecting the abandonments that have already occurred and assuming the relationship between the intention to abandon and the actual abandonment, it seems essential to develop a broad characterization of the students who intend to give up. At this point, with a sample of 267 Spanish students (48.7\% women; $\left.M_{a g e}=14.38 ; S D=1.09\right)$ of the $2 n d(n o=138)$ and the 3rd $(n=129)$ grade of Secondary Education, the incidence of repetition and academic performance on the intention to leave before completing compulsory secondary education are explored. The results of this work suggest that the probability of early abandonment is significantly higher among those who have repeated one or two courses in the high school than among those who have not repeated in that educational stage $(O R=8,769$, IC $95 \% 2,304-33,378 ; \mathrm{Q}=.79)$ and that the probability of quitting may also be higher among students who report lower than average academic performance than among those who report above-average performance ( $O R=4,336$, IC 95\%,968-19,421; $Q=.64)$. In light of our results, while not currently repeating or not having repeated in Primary Education could guarantee, to some extent, the intention to complete the studies, neither these factors nor the low ratings are established as direct predictors of the intention of early abandonment. In the light of these results, the personal and motivational profile of early school drop-out may continue to be explored.
\end{abstract}

Keywords: early school dropout; grade repetition; academic achievement; intention to drop out.

\section{INTRODUCCIÓN}

En palabras de Dekkers y Claassen (2001), el abandono escolar se define como el fracaso de un estudiante a la hora de completar su formación académica por diferentes causas. Específicamente, se entiende que una persona es partícipe de ese abandono prematuro de los estudios cuando los deja sin haber conseguido alguna formación más allá de la obligatoria. Se trataría por tanto de estudiantes de 18 a 24 años de edad que han obtenido como máximo el título de Educación Secundaria Obligatoria (ESO) o su equivalente en otros países europeos, y que no están cursando otros estudios (Serrano, Soler y Hernández, 2014).

A nivel europeo, el abandono escolar temprano ocupa una posición destacada dentro de las políticas educativas que se han adoptado. En esta línea, el marco estratégico Educación y Formación 2020 pretende la cooperación de los Estados miembros de la Unión Europea (UE) para así fomentar el desarrollo de sus propios sistemas de educación y formación, contribuyendo a su vez a disminuir el abandono escolar. Específicamente, en el año 2015 se puso de manifiesto que el abandono escolar prematuro se situaba en el 11.1\% pese a que el propósito de Europa 2020 fue situar dicho porcentaje por debajo del 10\% (Consejo y Comisión Europea, 2015). Por lo tanto, en el caso de Europa, y pese a que se ha producido una reducción generalizada de la tasa de abandono escolar temprano, siguen dándose tasas elevadas de abandono sobre todo en los países del sur mientras que las más bajas se dan en los países del norte.

Un buen ejemplo de ello está en España, Malta y Portugal donde uno de cada tres estudiantes abandona el sistema educativo prematuramente, encontrándose así importantes variaciones en la tasa de abandono escolar temprano que han ido desde un 37\% de abandono en el caso de Malta a un 4.3\% en Eslovaquia (González y de los Ríos, 2010; Nevala et al., 2011). Específicamente, el abandono prematuro de los estudios se da con mayor frecuencia entre los hombres que entre las mujeres, así como entre la población migrante y las minorías étnicas (Comisión Europea, 2011, 2017). 
Respecto a las diferencias entre géneros se ha afirmado que, generalmente, en España los hombres suelen tener peores calificaciones y suspenden más que las mujeres, dedicando estas más horas al estudio y adaptándose mejor a las normas y la vida del centro educativo (Cabrera-Pérez y Dahal, 2018).

Teniendo en cuenta la información proporcionada por el Instituto Nacional de Estadística (INE), en el 2016 nuestro país llegó a alcanzar una de las cifras de abandono escolar temprano más altas de todos los países de la UE, con un $23,2 \%$ en el caso de los hombres y un $15,4 \%$ respecto a las mujeres. Pese a que estas cifras han descendido en 2017 , bajando hasta un $21,5 \%$ en hombres y un $14,7 \%$ entre las mujeres según datos facilitados por el Ministerio de Educación, Cultura y Deporte (2018), actualmente el índice de abandono escolar en España sigue siendo uno de los más altos de la UE (Comisión Europea, 2017).

Partiendo de esta realidad, es lógico preguntarse por qué algunos estudiantes completan exitosamente su formación mientras que otros terminan abandonándola de manera prematura. La investigación ha reiterado que el abandono escolar no es simplemente una cuestión de género o de etnia, sino que se trata seguramente del resultado de un proceso gradual de desvinculación de la escuela, en el que influyen tanto factores geográficos como personales, económicos, familiares, del propio entorno educativo... que interactúan entre sí (Romero y Hernández, 2019). Debe tenerse presente que la motivación académica no se mantiene invariable a lo largo de la vida. Muchos niños y niñas comienzan en la escuela con una alta motivación intrínseca, pero esta tiende a disminuir a medida que avanzan en su educación dándose cambios especialmente importantes en los primeros años de la adolescencia, debido a que se trata de una etapa de cambio a nivel biológico, cognitivo y motivacional. Al llegar a la adolescencia las creencias, valoraciones y metas van cambiando y, poco a poco, van volviéndose relativamente estables, viéndose influidas por los éxitos y los fracasos repetidos que se van experimentando. Por lo tanto, el paso del colegio al instituto puede ir acompañado en muchos casos de una reducción importante del interés, de la curiosidad y del disfrute por aprender, así como de una disminución en las creencias de competencia, pudiendo llegar a derivar, en último término, en absentismo y abandono escolar temprano (Wigfield, Byrnes y Eccles, 2006; Wigfield y Cambria, 2010).

Todo un conjunto de factores personales (expectativas, autopercepciones, valoraciones, metas...) y de índole familiar (ambiente familiar y estilo parental percibido) repercuten en la decisión de abandonar los estudios prematuramente (Rumberger, 1995; Rumberger y Lim, 2008). En el ámbito escolar se han destacado factores como la escasa participación, el desinterés por los estudios, el bajo rendimiento académico o las tasas de suspensos, así como la repetición de curso o el absentismo, que podrían constituirse en factores asociados al abandono escolar temprano (Cairns, Cairns y Neckerman, 1989; De Witte y Csillag, 2014; Finn, 1989; González-Rodríguez, Vieira y Vidal, 2019; Janosz, Le Blanc, Boulerice y Tremblay, 2000; Mena, Fernández-Enguita y Rivière, 2010; Rodríguez y Guzmán, 2019).

Asumiendo que el abandono no se produce de manera repentina, sino que es el resultado de un proceso gradual que podría comenzar a manifestarse efectivamente en términos de bajo rendimiento, absentismo o repetición de curso, y en el que pueden influir factores como la pobreza, discapacidad, ambiente y configuración familiar, embarazo adolescente o el método de enseñanza empleado (Ngwakwe, 2014) en este trabajo abordamos la asociación entre el rendimiento medio y absoluto (suspensos/aprobados) y la repetición (en educación primaria o y ESO) y la intención de finalizar/no finalizar los estudios en secundaria obligatoria. Todo ello teniendo presente que el abandono temprano de la educación por parte de los jóvenes aumenta notablemente su riesgo de exclusión social (Tukundane, Minnaert, Zeelen y Kanyandago, 2015). 


\section{EL PAPEL DE LA REPETICIÓN Y EL RENDIMIENTO ACADÉMICO EN EL ABANDONO ESCOLAR TEMPRANO}

\section{OBJETIVOS DE LA INVESTIGACIÓN}

Con objeto de conocer las características de los estudiantes de secundaria que tienen intención de abandonar antes de completar sus estudios, en este trabajo se exploran las diferencias entre los estudiantes con intención de abandonar y los estudiantes con intención de continuar, en cuanto a las tasas de repetición y el rendimiento académico.

Atendiendo a la investigación previa se espera que los estudiantes con intención de abandono escolar tengan un rendimiento académico más bajo y mayores tasas de repetición que aquellos con intención de continuar con sus estudios (Bowers et al., 2013; Cairns et al., 1989; De Witte y Csillag, 2014; Finn, 1989; González-Rodríguez et al., 2019; Janosz et al., 2000; Mena et al., 2010; Rodríguez y Guzmán, 2019; Rumberger, 1995; Rumberger y Lim, 2008).

\section{PARTICIPANTES}

La muestra está integrada por 267 alumnos españoles (48.7\% mujeres; $\mathrm{M}_{\mathrm{age}}=14.38 ; S D=1.09$ ) que cursaban $2^{0}(n=138)$ y $3^{\circ}(n=129)$ de ESO.

\section{METODOLOGÍA}

\section{Análisis de datos}

Se exploran en primer lugar las diferencias de proporciones en repetición y rendimiento académico entre estudiantes con intención de abandono y estudiantes sin intención de abandono (intención de continuar) empleando $\chi 20$ prueba exacta de Fisher según el caso ${ }^{1}$. Con objeto de estimar la probabilidad de abandono antes de finalizar la ESO, frente a la intención de continuar estudiando, en presencia de alto/bajo rendimiento, rendimiento superior/inferior a la media, repetición/no repetición EP, repetición/no repetición en ESO, se emplea el modelado logístico. La probabilidad es estimada mediante el estadístico denominado odd ratio (OR). Dado que la interpretación de la odd ratio como probabilidad puede resultar confusa, a efectos de interpretación, se incorpora el índice $Q$ de Yule como medida de asociación o co-ocurrencia binaria mediante transformación de los odds ratios en una escala que va de -1 a 1 , donde 0 indica independencia. La categorización de la variable continua rendimiento se realiza con respecto al aprobado -alto/bajo rendimiento- y respecto a la media del grupo -rendimiento superior/inferior a la media-.

\section{Variables}

Para conocer la intención de abandono de la muestra antes de finalizar la ESO se ha utilizado el ítem:

"Voy a dejar los estudios antes de terminar la ESO" empleando una medida dicotómica (En Desacuerdo = 0; De acuerdo =1), del tal modo que el acuerdo (1) con la afirmación se interpreta como intención de abandonar y el desacuerdo (0) como intención de no abandono o intención de continuar estudiando.

Las variables de repetición se evaluaron dicotomizando los ítems:

¿Cuántos cursos has repetido en Primaria? No (0) Si (1, 2 o 3$)$

¿Cuántos cursos has repetido en ESO? No No (0) Si (1, 2 o 3$)$

Se calculó el rendimiento medio a partir de las calificaciones habituales en una serie de materias: ¿Qué nota sueles sacar en: (Matemáticas, Lengua Española, Biología, Geografía e Historia, Inglés -idiomas- y Lingua Galega) sobre una escala de 1 a 10. La variable rendimiento absoluto se evaluó dicotomizando el ítem rendimiento medio en base al aprobado: bajo $(\geq 5)$ / alto $(<5)$ y el rendimiento relativo en base a la media del grupo.

Todos estos análisis se hicieron a través del paquete estadístico SPSS 22.0. 


\section{RESULTADOS ALCANZADOS}

El análisis de resultados nos permite informar de que solo el $4.5 \%$ de nuestra muestra tiene intención de abandonar. El 88.3\% de los estudiantes con intención de abandonar antes de finalizar los estudios obligatorios son varones, y la intención de abandono puede ser ligeramente más elevada entre los estudiantes que están cursando $2^{\circ}$ de la ESO (5.1\%) que entre quienes cursan 3ํㅜ (3.9\%).

\section{Repetición e intención de abandono}

Los análisis bivariados revelan diferencias significativas en intención de abandonar y continuar en función de la repetición en ESO ( $p<.01$ en pruebas exactas de Fisher). Mientras que las diferencias en la proporción de repetidores en EP con intención de abandono y sin intención de abandono no alcanzan significatividad, el 75\% de los estudiantes con intención de abandonar sus estudios antes de finalizar la educación secundaria son estudiantes que han repetido al menos un curso en ESO (Véase Tabla 1).

\section{Tabla 1}

Porcentaje y frecuencia de repetidores con intención de abandonar y sin intención de abandonar (intención de continuar)

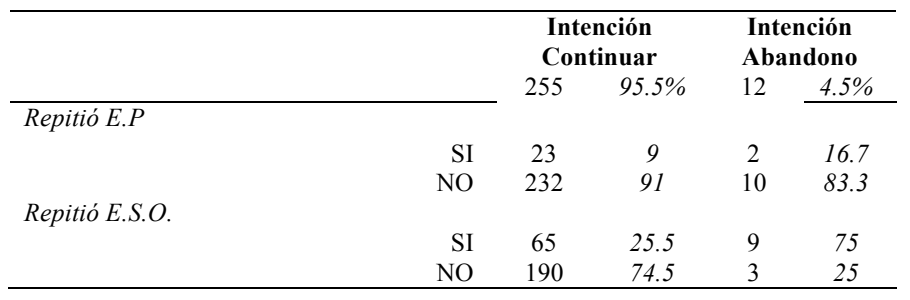

Los resultados de este trabajo sugieren que la probabilidad de que se dé una intención de abandono temprano es significativamente más alta entre quienes han repetido uno o dos cursos en la ESO que entre aquellos que no han repetido en ESO (OR=8,769, IC 95\% 2.304-33.378; Q=.79). La repetición en EP no parece indicador relevante de la intención de abandono temprano (Véase Tabla 1)

\section{Rendimiento académico e intención de abandono}

Los análisis bivariados sugieren la existencia de diferencias en las proporciones de sujetos con intención de abandono en función del rendimiento respecto a la media $(p<.05$ en pruebas exactas de Fisher), pero no en función del rendimiento bajo/alto. Tal y como puede apreciarse en la Tabla 2 , el $83.3 \%$ de los estudiantes con intención de abandonar sus estudios antes de finalizar la ESO son estudiantes con un rendimiento inferior al de la media.

Tabla 2

Porcentaje y frecuencia de estudiantes con intención de abandonar y sin intención de abandonar (intención de continuar) en función del rendimiento

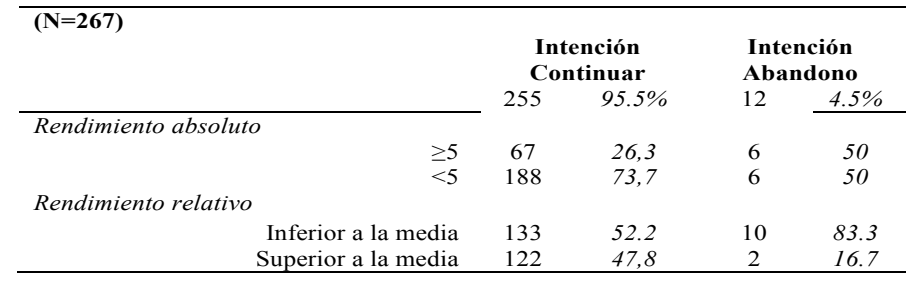




\section{EL PAPEL DE LA REPETICIÓN Y EL RENDIMIENTO ACADÉMICO EN EL ABANDONO ESCOLAR TEMPRANO}

Atendiendo a nuestros resultados efectivamente la probabilidad de abandonar puede ser más elevada entre los estudiantes que informan de rendimiento académico inferior a la media que entre quienes informan de rendimiento superior a la media ( $O R=4.336$; IC $95 \% .968-19.421 ; Q=.64)$.

\section{DISCUSIÓN}

Haber repetido en educación secundaria parece ser un factor de influencia en el abandono escolar temprano. Efectivamente, este trabajo sugiere que aquellos alumnos/as que han repetido al menos un curso en la ESO son más propensos a dejar los estudios que aquellos que nunca han repetido en esta etapa, de modo que la repetición en educación secundaria sí podría aumentar la probabilidad de abandonar (Alexander, Entwisle y Dauber, 2003).

Sin embargo, no se ha encontrado la misma relación entre repetición en educación primaria y la probabilidad de abandono, de manera que haber repetido en esta etapa no tendría un peso significativo en el futuro abandono escolar. Estos resultados contradicen la afirmación de que repetir en educación primaria aumentaría la probabilidad de abandono escolar temprano, especialmente entre Ios estudiantes en riesgo por causas personales y sociofamiliares (Hughes, Cao, West, Smith y Cerda, 2017; Stearns, Moller, Blau, Potochnick, 2007). En consecuencia, nuestros hallazgos apuntan a que la repetición en primaria no parece ser relevante para la intención de abandono.

Respecto al rendimiento, se ha señalado que el bajo rendimiento académico es uno de los factores que hace al alumno/a más vulnerable ante la posibilidad de abandonar (Cairns et al., 1989; Janosz et al., 2000). Los resultados obtenidos apuntan a que cuando el rendimiento es inferior a la media del grupo en el que se encuentra el alumno/a es más probable que este abandone antes de terminar la ESO. De este modo, el hecho de tener un rendimiento objetivamente alto o bajo no parece ser tan importante como la comparación con el nivel del grupo de referencia.

Destaca también el hecho de que la mayor parte de los estudiantes con intención de abandono temprano son hombres, siendo esta una de las principales características de la mayoría del alumnado con calificaciones más bajas y de perfil repetidor (Cabrera-Pérez y Dahal, 2018; Méndez y Cerezo, 2018).

\section{CONCLUSIONES}

La mayoría de los modelos que han abordado la explicación del abandono escolar temprano han asumido este como un proceso complejo que está influido por múltiples y diversas condiciones.

Así la repetición en ESO no parece mejorar el rendimiento académico del alumnado, sino que, contrariamente, aumentaría la frustración y la desconexión respecto a la escuela favoreciendo la deserción (Manacorda, 2012; Roderick, 1995), lejos de beneficiar a los alumnos que hacen uso de ella en el instituto. Los resultados derivados de esta investigación con estudiantes de secundaria llevan a pensar nuevamente en la influencia significativa de la repetición y del rendimiento como factores que influyen en la probabilidad de abandonar los estudios, aumentando las posibilidades de abandono temprano siempre y cuando esa repetición se produzca en la etapa de secundaria y cuando el rendimiento académico sea inferior a la media del grupo en el que se encuentra el alumno/a. Recordemos, por tanto, que no se ha encontrado relación entre la repetición en primaria y el aumento de la probabilidad de abandono escolar, ni entre el rendimiento objetivamente bajo y el abandono.

Sin olvidar que los estudiantes varones han tenido una mayor intención de abandono que sus compañeras de aula, resultado que encaja con los planteamientos iniciales de este trabajo.

No obstante, la familia también juega un papel relevante sobre el desempeño del estudiante. Cuando este crece en un ambiente familiar estable en el que se le supervisa adecuadamente, cre- 
yendo en su capacidad para tener éxito e intentando que se sienta escuchado, la probabilidad de abandono escolar se reduce (Astone y McLanahan, 1991).

A su vez, la participación escolar es un elemento de gran poder sobre los resultados académicos (Connell, 1990). Como explica Finn (1993), aquellos estudiantes que no participan activamente en las actividades relacionadas con la escuela, o que no se sienten identificados con el entorno escolar, tienen un mayor riesgo de abandono, puesto que la participación educativa y el rendimiento académico están positivamente relacionados independientemente del género, de la etnia y del nivel socioeconómico.

Por ello, la existencia de un clima escolar positivo y la puesta en marcha de políticas y prácticas educativas que promuevan el compromiso reducen notablemente el abandono (Rumberger y Lim, 2008). Consecuentemente, los centros educativos deberían entenderse como redes de apoyo a los estudiantes en riesgo, atendiendo a sus necesidades e intereses y potenciando sus fortalezas para fomentar el sentimiento de pertenencia y la participación (Finn, 1989; Ngwakwe, 2014; Wehlage, Rutter, Smith, Lesko y Fernández, 1989), realizando a su vez intervenciones educativas que partan de una metodología flexible y holística para prevenir la deserción (Tukundane et al., 2015).

\section{REFERENCIAS BIBLIOGRÁFICAS}

Alexander, K.L., Entwisle, D.R. y Dauber, S.L. (2003). On the success of failure: A reassessment of the effects of retention in the primary school grades. New York: Cambridge University Press.

Astone, N.M. y McLanahan, S.S. (1991). Family Structure, Parental Practices and High School Completion. American Sociological Review, 56(3), 309-320.

Bowers, A.J., Sprott, R. y Taff, S.A. (2013). Do we know who will drop out?: A review of the predictors of dropping out of high school: Precision, sensitivity, and specificity. The High School Journal, 96(2), 77-100.

Cabrera-Pérez, L. y Dahal, T. (2018). El abandono educativo temprano: ¿una cuestión de género? Comparando España y Nepal. XVI Congreso Nacional Educación Comparada Tenerife.

Cairns, R.B., Cairns, B.D. y Neckerman, H.J. (1989). Early School Dropout: Configurations and Determinants. Child Development, 60(6), 1437-1452.

Comisión Europea (2011). Abordar el abandono escolar prematuro: una contribución clave a la agenda Europa 2020. Comunicación de la Comisión al Parlamento Europeo, al Consejo, al Comité Económico y Social Europeo y al Comité de las Regiones.

Comisión Europea (2017). Monitor de la Educación y la Formación de 2017. España. Dirección General de Educación y Cultura.

Connell, J.P. (1990). Context, Self and Action: A motivational Analysis of Self-System Processes Across the Life Span. En D. Cicchetti y M. Beeghly (Eds.), The Self in Transition: Infancy to Childhood (pp. 61-97). Chicago: University of Chicago Press.

Consejo y Comisión Europea (2015). Informe conjunto de 2015 del Consejo y de la Comisión sobre la aplicación del marco estratégico para la cooperación europea en el ámbito de la educación y la formación (ET 2020). Nuevas prioridades para la cooperación europea en educación y formación. Diario Oficial de la Unión Europea.

Dekkers, H. y Claassen, A. (2001). Dropouts: Disadvantaged by Definition? A Study of the Perspective of Very Early School Leavers. Studies in Educational Evaluation, 27(4), 341-354.

De Witte, K. y Csillag, M. (2014). Does anybody notice? On the impact of improved truancy reporting on school dropout. Education Economics, 22(6), 549-568.

Finn, J.D. (1993). School Engagement \& Students at Risk. Washington, DC: National Center for Education Statistics (ED). 


\section{EL PAPEL DE LA REPETICIÓN Y EL RENDIMIENTO ACADÉMICO EN EL ABANDONO ESCOLAR TEMPRANO}

Finn, J.D. (1989). Withdrawing from school. Review of Educational Research, 59(2), 117-142.

González-Rodríguez, D., Vieira, M.J. y Vidal, J. (2019). Factors that influence early school leaving: a comprehensive model. Educational Research, 61(2), 214-230.

González, V.M. y de los Ríos, S. (2010). Estrategia Europa 2020: mujer, educación y empleo. CLM Economía, 17, 231-261.

Hughes, J.N., Cao, Q., West, S.G., Smith, P.A. y Cerda, C. (2017). Effect of retention in elementary grades on dropping out of school early. Journal of School Psychology, 65, 11-27.

Instituto Nacional de Estadística. En INE: productos y servicios/ publicaciones/ publicaciones de descarga gratuita/ mujeres y hombres en España/ educación/ abandono temprano de la educación-formación. Recuperado el 13 de junio de 2018, de http://www.ine.es/ss/Satellite?c=INESeccion_C\&cid=1259925480602\&p=1254735110672\&pag ename=ProductosYServicios\%2FPYSLayout

Janosz, M., Le Blanc, M., Boulerice, B. y Tremblay, R.E. (2000). Predicting different types of school dropouts: A typological approach with two Iongitudinal samples. Journal of Educational Psychology, 92(1), 171-190.

Manacorda, M. (2012). The cost of grade retention. The Review of Economics and Statistics, 94(2), 596-606.

Mena, L., Fernández-Enguita, M. y Rivière, J. (2010). Desenganchados de la educación: procesos, experiencias, motivaciones y estrategias del abandono y del fracaso escolar. Revista de Educación, núm. extraordinario, 119-146.

Méndez, I. y Cerezo, F. (2018). La repetición escolar en educación secundaria y factores de riesgo asociados. Educación XXI, 21(1), 41-61.

Ministerio de Educación, Cultura y Deporte (2018). En MECD: prensa/ actualidad/ abandono escolar temprano. Recuperado el 13 de junio de 2018, de https://www.mecd.gob.es/prensamecd/actualidad/2017/07/2017027-abandono.html

Nevala, A.M., Hawley, J., Stokes, D., Slater, K., Souto-Otero, M., Santos, R., et al. (2011). Reducing early school leaving in the EU. Directorate General for Internal Policies, European Parliament.

Ngwakwe, C.C. (2014). A review of causes of school dropouts in brics countries: A South African case. Corporate Ownership \& Control, 12(1), 911-914.

Roderick, M. (1995). Grade Retention and School Dropout: Policy Debate and Research Questions. The Research Bulletin, 15, 88-92.

Rodríguez, D. y Guzmán, R. (2019). Rendimiento académico de adolescentes declarados en situación de riesgo. Revista de Investigación Educativa, 37(1), 147-162.

Romero, E. y Hernández, M. (2019). Análisis de las causas endógenas y exógenas del abandono escolar temprano: una investigación cualitativa. Educación XXI, 22(1), 263-293.

Rumberger, R.W. (1995). Dropping out of middle school: A multilevel analysis of students and schools. American Educational Research Journal, 32(3), 583-625.

Rumberger, R.W. y Lim, S.A. (2008). Why Students Drop Out of School: A Review of 25 Years of Research. University of California, Santa Barbara: California Dropout Research Project.

Serrano, L., Soler, A. y Hernández, L. (2014). El abandono educativo temprano: análisis del caso español. Instituto Valenciano de Investigaciones Económicas (IVIE), Ministerio de Educación, Cultura y Deporte.

Stearns, E., Moller, S., Blau, J. y Potochnick, S. (2007). Staying back and dropping out: The relationship between grade retention and school dropout. Sociology of Education, 80(3), 210-240.

Tukundane, C., Minnaert, A., Zeelen, J. y Kanyandago, P. (2015). A review of enabling factors in support intervention programmes for early school leavers: What are the implications for SubSaharan Africa? Children and Youth Services Review, 52, 54-62. 
Wehlage, G.G., Rutter, R.A., Smith, G.A., Lesko, N.L. y Fernández, R.R. (1989). Reducing the Risk: Schools as Communities of Support. Philadelphia, PA: The Falmer Press.

Wigfield, A., Byrnes, J.P. y Eccles, J.S. (2006). Development during early and middle adolescence. En P.A. Alexander y P.H. Winne (Eds.), Handbook of Educational Psychology. Second Edition (pp. 87-113). New York, NY: Routledge.

Wigfield, A. y Cambria, J. (2010). Achievement motivation. En I.B. Weiner y W.E. Craighead (Eds.), The Corsini Encyclopedia of Psychology. Fourth Edition (Vol. 1, pp. 14-15). New Jersey: John Wiley \& Sons.

1 Cuando más del $20 \%$ de las celdas tienen frecuencias esperadas $<5$ se empleará la prueba exacta de Fisher en lugar de un método de aproximación. 
